\title{
Advances in cardiac rehabilitation: cardiac rehabilitation after transcatheter aortic valve implantation
}

\author{
Sarah Eichler'1, Heinz Völler1,2 \\ 1 Center of Rehabilitation Research, University of Potsdam \\ 2 Department of Cardiology, Klinik am See, Rüdersdorf, Germany
}

\begin{abstract}
For more than a decade, transcatheter aortic valve implantation (TAVI) has become a promising treatment modality for patients with severe aortic stenosis and a high surgical risk. To improve exercise capacity and quality of life, cardiac rehabilitation (CR) including physical activity is a well-established treatment for patients after cardiac valve surgery. First studies have shown that CR could also be a helpful tool to maintain independency for activities of daily living and participation in socio-cultural life in patients after TAVI. Strength and balance training are important parts of physical activity in octogenarians and have been investigated in healthy older adults in several studies, but need to be widened and investigated for TAVI patients. Hence, for this older patient group, there are more prospective multicentre studies needed.
\end{abstract}

\section{Introduction}

Due to the demographic change and an aging population, the prevalence of the most frequent valve disease, aortic stenosis (AS), is still rising $[1,2]$.

For patients with severe AS and a prohibitive surgical risk, transcatheter aortic valve implantation (TAVI) has developed as a golden standard [3-5]. First performed in a human body in 2002 [6], the procedure is rapidly growing in utilization. A total of 48,353 TAVI procedures have been performed in Germany with a 20 -fold increase since

Corresponding author: Dr. Heinz Völler, University of Potsdam, Center of Rehabilitation Research, Am Neuen Palais 10, Haus 12, 14469 Potsdam, Germany. Tel. +49.331.9774062 - Fax +49.331.9774081.

E-mail: heinz.voeller@uni-potsdam.de

Key words: Aortic stenosis; exercise capacity; quality of life; cardiac rehabilitation; TAVI.

Received for publication: 19 April 2016

Accepted for publication: 04 July 2016

CC Copyright S. Eichler and H. Völler, 2016

Tipografia PI-ME Editrice, Italy

Monaldi Archives for Chest Disease Cardiac Series 2016; 86:758

doi: 10.4081/monaldi.2016.758

This article is distributed under the terms of the Creative Commons Attribution Noncommercial License (by-nc 4.0) which permits any noncommercial use, distribution, and reproduction in any medium, provided the original author(s) and source are credited.
2008 [7]. Several clinical trials and registries have shown the advantages and the procedural success concerning mid- to long-term outcomes with an improved survival rate compared to the standard therapy [3,8-10].

\section{Cardiac rehabilitation after TAVI}

For the improvement of exercise capacity, quality of life and morbidity, cardiac rehabilitation (CR) including physical activity is a wellestablished treatment for patients after cardiac valve surgery [11-13]. As TAVI starts to get more common nowadays and numbers are steadily increasing, this relatively new patient group also becomes more present in CR. Little is known about the efficacy of CR in TAVI patients as there exist only few studies about CR in this high-risk patient group in the older age, often having several comorbidities. Russo et al. [14] investigated the efficacy regarding functional capacity and additionally the safety of CR in TAVI patients and patients after surgical aortic valve replacement (sAVR). Though having many comorbidities, no major complications occurred in TAVI patients. In the overall patient group, a significant increase the 6-minute walk test (6MWT) could be achieved. The authors showed that a supervised, short-term, exercisebased CR program is feasible, safe and effective in these octogenerians. Völler et al. [15] could also show a benefit in functional status in patients after TAVI undergoing CR. Measured by the 6MWT and a bicycle stress test, the patients reached a significant longer walking distance as well as a significant higher exercise capacity at discharge of the three-week inpatient structured rehabilitation program, that consisted of individualised physical training, patient education and psychological support [16]. As the valve academic research consortium-2 consensus document (VARC-2) [17] underlines the necessity of frailty as a multicomponent factor with the criteria slowness, weakness, exhaustion, wasting and malnutrition, poor endurance and inactivity as well as a loss of independence, evidence for these factors is needed. Zanettini et al. [18] could show that most TAVI patients obtained a significant improvement of an in-hospital CR program concerning functional status, quality of life and autonomy, which even remained constant during mid-term follow-up.

The first results of CR in TAVI patients show that CR can be a helpful tool to maintain independency for activities of daily living and participation in socio-cultural life, but still there are more prospective multicentre studies including geriatric assessments, such as frailty, needed to have enough evidence for the improvement and the prognosis of this high-risk and multimorbid patient group. Preliminary results of a prospective multicenter registry with a geriatric pre-interventional assessment according to Schoenenberger et al. [19], which was repeated at admission and discharge of inpatient $\mathrm{CR}$, revealed that frailty could be a transient status in the majority of patients. Compared to the preinterventional measurement, where $47.3 \%$ had a positive Frailty-Index, 
at discharge of inpatient CR only $33.3 \%$ of the TAVI patients were still considered as frail [20].

\section{Final remarks}

Other factors, e.g. the importance of fall prevention or strength training, have been investigated in healthy older adults in several studies [21-23], but need to be widened and investigated for TAVI patients. Cardiac rehabilitation researchers need to investigate and implement programs and protocols that include physical training with a combination of balance training in dual- and multi-tasking situations and strength training, mainly focusing on the lower extremities.

\section{References}

1. Carabello BA, Paulus WJ. Aortic stenosis. Lancet 2009;373:956-66.

2. Nishimura RA, Otto CM, Bonow R0, et al. 2014 AHA/ACC Guideline for the management of patients with valvular heart disease: a report of the American College of Cardiology/American Heart Association Task Force on Practice Guidelines. Circulation 2014;129:e521-643.

3. Leon MB, Smith CR, MAck M, et al. Transcatheter aortic-valve implantation for aortic stenosis in patients who cannot undergo surgery. N Engl J Med 2010;363:1597-607.

4. Vahanian A, Alfieri 0, Andreotti F, et al. Guidelines on the management of valvular heart disease (version 2012): the Joint Task Force on the Management of Valvular Heart Disease of the European Society of Cardiology (ESC) and the European Association for Cardio-Thoracic Surgery (EACTS). Eur J Cardiothorac Surg 2012;42:S1-44.

5. Généreux P, Head SJ, Wood DA, et al. Transcatheter aortic valve implantation 10-year anniversary: review of current evidence and clinical implications. Eur Heart J 2012;33:2388-98.

6. Cribier A, Eltchaninoff H, Bash A, et al. Percutaneous transcatheter implantation of an aortic valve prosthesis for calcific aortic stenosis: first human case description. Circulation 2002;106:3006-8.

7. Eggebrecht H, Mehta RH. Transcatheter aortic valve implantation (TAVI) in Germany 2008-2014: on its way to standard therapy for aortic valve stenosis in the elderly? EuroIntervention 2016;11: 1029-33.

8. Smith CR, Leon MB, Mack MJ, et al. Transcatheter versus surgical aortic-valve replacement in high-risk patients. $\mathrm{N}$ Engl $\mathrm{J}$ Med 2011;364:2187-98.

9. Moat NE, Ludman P, de Belder MA, et al. Long-term outcomes after transcatheter aortic valve implantation in high-risk patients with severe aortic stenosis: the U.K. TAVI (United Kingdom Transcatheter Aortic Valve Implantation) Registry. J Am Coll Cardiol 2011;58:2130-8.

10. Ussia GP, Barbanti M, Petronio AS, et al. Transcatheter aortic valve implantation: 3-year outcomes of self-expanding CoreValve prosthesis. Eur Heart J 2012;33:969-76.

11. Butchart EG, Gohlke-Bärwolf C, Antunes MJ, et al. Recommendations for the management of patients after heart valve surgery. Eur Heart J 2005;26:2463-71.

12. Horstkotte D, Lengyel M, Mistiaen WP, et al. Recommendations for post-discharge patient follow up after cardiac valve interventions: a position paper. J Heart Valve Dis 2007;16:575-89.

13. Kiel MK. Cardiac rehabilitation after heart valve surgery. PM R 2011;3:962-7.

14. Russo N, Compostella L, Tarantini G, et al. Cardiac rehabilitation after transcatheter versus surgical prosthetic valve implantation for aortic stenosis in the elderly. Eur J Prev Cardiol 2014;21:1341-8.

15. Voller H, Salzwedel A, Nitardy A, et al. Effect of cardiac rehabilitation on functional and emotional status in patients after transcatheter aortic-valve implantation. Eur J Prev Cardiol 2015;22:568-74.

16. Karoff M, Held K, Bjarnason-Wehrens B. Cardiac rehabilitation in Germany. Eur J Cardiovasc Prev Rehabil 2007;14:18-27.

17. Kappetein AP, Head SJ, Généreux P, et al. Updated standardized endpoint definitions for transcatheter aortic valve implantation: the Valve Academic Research Consortium-2 consensus document. J Thorac Cardiovasc Surg 2013;145:6-23.

18. Zanettini R, Gatto G, Mori I, et al. Cardiac rehabilitation and midterm follow-up after transcatheter aortic valve implantation. J Geriatr Cardiol 2014;11:279-85.

19. Schoenenberger AW, Stortecky S, Neumann S, et al. Predictors of functional decline in elderly patients undergoing transcatheter aortic valve implantation (TAVI). Eur Heart J 2013;34:684-92.

20. Völler H, Eichler S, Harnath A, et al. Case management in patients after TAVI: are frailty and exercise capacity predictors for decision making process? Proceedings European Society of Cardiology Congr., London, 2015: Abstract No. 81966.

21. Borde R, Hortobagyi T, Granacher U. Dose-response relationships of resistance training in healthy old adults: a systematic review and meta-analysis. Sports Med 2015;45:1693-720.

22. Granacher U, Gollhofer A, Hortobágyi T, et al. The importance of trunk muscle strength for balance, functional performance, and fall prevention in seniors: a systematic review. Sports Med 2013;43:627-41.

23. Granacher U, Muehlbauer T, Gschwind YJ, et al. [Assessment and training of strength and balance for fall prevention in the elderly: recommendations of an interdisciplinary expert panel].[Article in German]. Z Gerontol Geriatr 2014;47:513-26. 